\title{
Biomedical diagnostics enabled by integrated organic and printed electronics
}

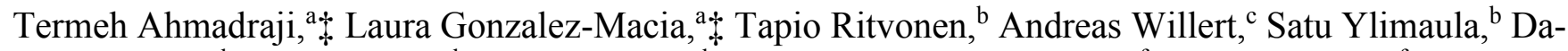 \\ vid Donaghy, ${ }^{\mathrm{d}}$ Saara Tuurala, ${ }^{\mathrm{b}}$ Mika Suhonen, ${ }^{\mathrm{b}}$ Dave Smart, ${ }^{\mathrm{e}}$ Aoife Morrin, ${ }^{\mathrm{f}}$ Vitaly Efremov, ${ }^{\mathrm{f}}$ Reinhard \\ R. Baumann, ${ }^{\mathrm{g}}$ Munira Raja, ${ }^{\mathrm{d}}$ Antti Kemppainen ${ }^{\mathrm{b}}$ and Anthony J. Killard ${ }^{\mathrm{a}, *}$

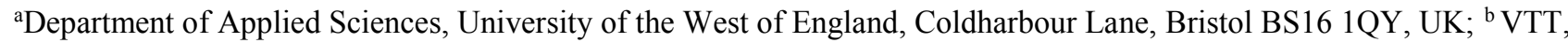 \\ P.O. Box 1000, FI-02044 VTT, Finland; ' Fraunhofer Institute for Electronic Nanosystems ENAS, Technologie-Campus 3 , \\ 09126 Chemnitz, Germany; ${ }^{d}$ Electrical Engineering and Electronics, University of Liverpool, Brownlow Hill, Liverpool L69 \\ 7ZX, UK; ${ }^{\mathrm{e}}$ Smart Dx Consulting Ltd, 4 Smeaton Park, Moira BT67 0NF, UK; ${ }^{\mathrm{f}}$ School of Chemical Sciences and National \\ Centre for Sensor Research, Dublin City University, Dublin 9, Ireland.; ${ }^{\mathrm{g}}$ Department Digital Printing and Imaging Technolo- \\ gies, Technische Universität Chemnitz,Reichenhainer Str. 70, 09126 Chemnitz, Germany.
}

\begin{abstract}
Organic and printed electronics integration has the potential to revolutionise many technologies, including biomedical diagnostics. This work demonstrates the successful integration of multiple printed electronic functionalities into a single device capable of the measurement of hydrogen peroxide, and total cholesterol. The single-use device employed printed electrochemical sensors for hydrogen peroxide electroreduction integrated with printed electrochromic display and battery. The system was driven by a conventional electronic circuit designed to illustrate the complete integration of silicon ICs via pick and place, or using organic electronic circuits. The device was capable of measuring $8 \mu \mathrm{L}$ samples of both hydrogen peroxide $\left(0\right.$ to $\left.5 \mathrm{mM}^{2} 2.72 \times 10^{-6} \mathrm{~A} . \mathrm{mM}^{-1}\right)$ and total cholesterol in serum from 0 to $9 \mathrm{mM}\left(1.34 \times 10^{-8} \mathrm{~A} \cdot \mathrm{mM}^{-1}, \mathrm{r}^{2}=0.99, \mathrm{RSD}<10 \%, \mathrm{n}=3\right)$ which was output on a semi-quantitative linear bar display. The device could operate for 10 minutes via a printed battery and display the result for many hours or days. A mobile phone 'app' was also capable of reading the test result and transmitting this to a remote health care provider. Such a technology could allow improved management of conditions such as hypercholesterolemia.
\end{abstract}

Printed electronics is being hailed as a technological revolution, equal in importance to the emergence of microelectronics over 50 years ago. The combined qualities of print-processable organic, inorganic and hybrid (semi)conductive materials which can be deposited onto flexible polymeric substrates using a range of additive, high throughput printing methodologies offer the prospect of low cost mass production capability and the potential for unprecedented levels of technological integration. ${ }^{1-3}$ This potent combination is transformational in the ways in which we use electronic technologies, and further embed them into our daily lives. Such technologies are already being integrated into many everyday appliances such as organic light emitting diode (OLED) displays on TVs, tablets and phones, integrated lighting on product packaging, vehicles and in the built environment, printed RFID tags for object tracking and many more besides. ${ }^{1,4,5}$

One area that has great potential for its integration with organic and printed electronics is that of sensors and diagnostics. In fact, sensors - electrochemical sensors at least - could be regarded as far ahead of their time in the development of printed electronics. Printed electrochemical sensor strips have been with us for some 20 years now for application in blood glucose monitoring and have featured the use of conductive inks printed onto planar substrates to form printed electrodes as the basis of miniaturized electrochemical cells. ${ }^{6,7}$ Other reagents such as enzymes and mediators have also been effectively integrated and deposited using screen printing techniques. However, to perform the rest of the measurement, the printed sensor has always been dependent on a number of traditional elements which include displays, batteries and silicon circuitry external to the sensor in the form of a meter. In addition, the cost and complexity of the meter components dictates that they are used repeatedly and require maintenance and calibration.

The next stage of technological evolution is to fully integrate all of these components into a single monolithic device through the medium of organic and printed electronics. However, to achieve this, several technological hurdles have to be overcome. Most of these relate to the types and range of materials that must be amenable to integrated fabrication processing methods. This means having the appropriate functionality, print processability and compatibility with other materials and other processes used during manufacturing. This results in a significant complexity challenge to effectively combine all these functionalities into operational devices.

In this work, we demonstrate the fabrication and integration of printed electrochemical sensors, electrochromic display and battery onto a single polymeric substrate. The integrated sensor system (SIMS) combines various organic, flexible and printed electronics (OFPE) technologies with potential for application in diagnostics and other areas. In addition, we demonstrate its interfacing with simplified conventional electronic circuits as a prelude for future full integration of pick and place silicon chips or organic electronic circuits. 


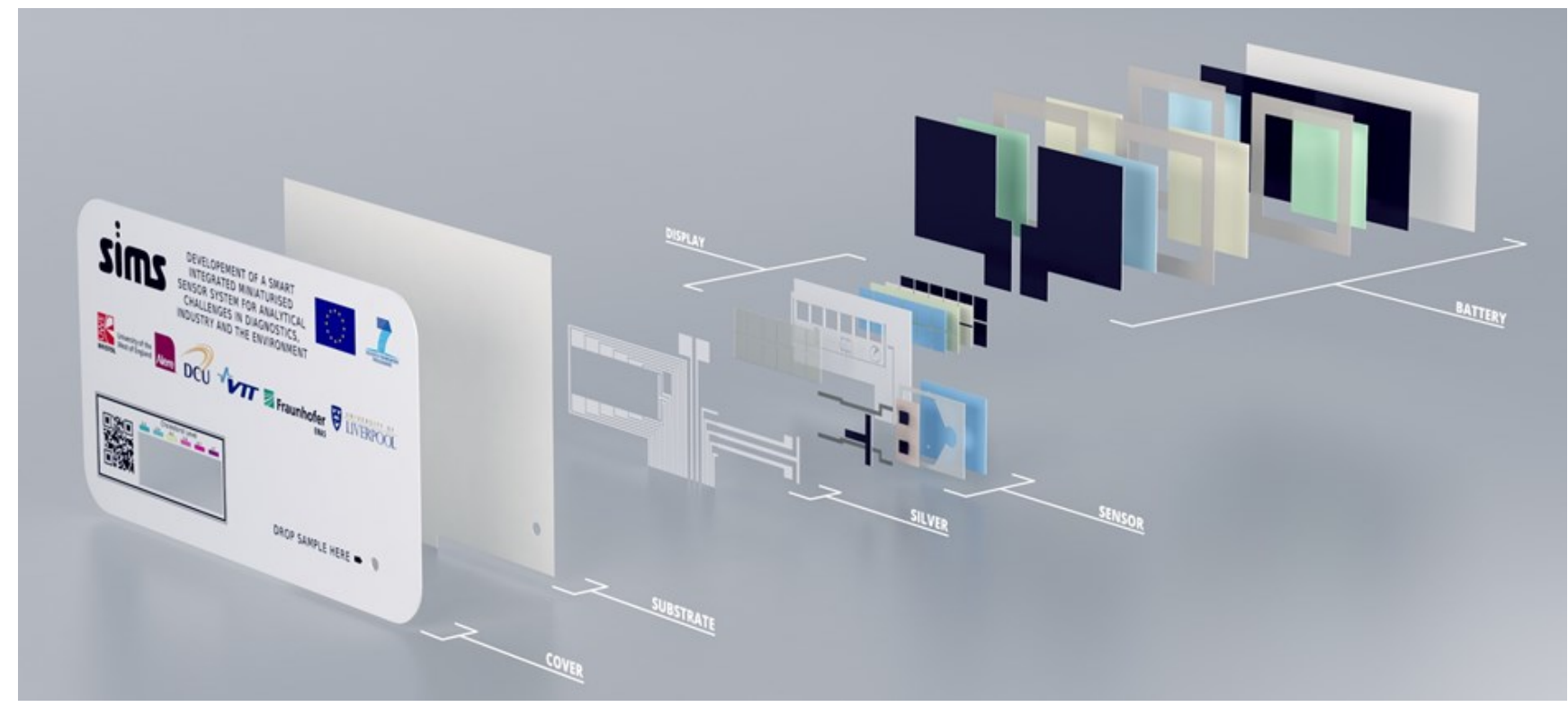

Figure 1. Complete layer stack of the SIMS device. A printed card device cover with display window, QR code, printed cholesterol level and color coding. Transparent polymer substrate layer based on polyethylene naphthalate foil for high dimensional stability during processing. Common silver layer for interconnects for sensor, display and battery. Display, battery and sensor layer stacks are shown in detail in Figs. 2, 3 and 5.

\section{EXPERIMENTAL SECTION}

Devices were fabricated on polyethylene napthalate $(\mathrm{PEN})$ substrates of $100 \mathrm{~mm} \times 80 \mathrm{~mm}$ (Teonex Q65FA) (Teijin Dupont Films), modified with indium-doped tin oxide (ITO). Q65FA was chosen for its excellent characteristics of process compatibility and dimensional stability. The ITO display current collector was patterned by etching. Silver tracks were deposited using screen printing of Electrodag PF-410 (Henkel) on a DEK 248 screen printer (DEK, UK). All display components were deposited using screen printing of the electrochromic ink set $(\mathrm{Na}-$ noChromics ${ }^{\mathrm{TM}}$ Print Trial Kit TKG-3.1) developed by NTera (Dublin, Ireland). Dual three electrode electrochemical cells were fabricated using screen printing. Silver (PF-410) was used as the conductor and working electrodes, while $\mathrm{Ag} / \mathrm{AgCl}$ Electrodag $6037 \mathrm{SS}$ and carbon Electrodag $6017 \mathrm{SS}$ (Henkel) were used as reference and common counter electrodes, respectively. Working electrode area was $3 \mathrm{~mm} \times 3 \mathrm{~mm}$.

Batteries were fabricated using a combination of screen printing, gluing and lamination. The current collectors were fabricated using screen printing of PF-407C (Henkel). Anodes and cathodes employed manganese dioxide and zinc-based inks, respectively. The anode and cathode were laminated together employing double-sided glue tape with polymer separator layer and $\mathrm{ZnCl}_{2}$ gel electrolyte.

For the demonstration of the measurement of $\mathrm{H}_{2} \mathrm{O}_{2}$, electrodes were modified with the inkjet-printed deposition of Triton X-100 $(0.25 \% \mathrm{v} / \mathrm{v})$ in PBS as electrocatalyst using a Dimatix DMP 2831 inkjet printer, with an estimated total volume of $952 \mathrm{~nL}$. For the measurement of TC, four inkjet-printed layers of cholesterol esterase (ChEs) $(1.04 \mathrm{kU} / \mathrm{mL})$ and cholesterol oxidase (ChOx) $(0.39 \mathrm{kU} / \mathrm{mL})$ in $0.25 \%(\mathrm{v} / \mathrm{v})$ Triton X-100, and stabilisers $0.2 \%(\mathrm{w} / \mathrm{v})$ polyvinylpyrrolidone and poly(diallyldimethylammonium chloride) in PBS were deposited to a total volume of $1.5 \mathrm{~mL}$ and allowed to dry under ambient conditions. Cholesteryl acetate stock solution $(500 \mathrm{mg} / \mathrm{dL})$ was prepared in $\mathrm{PBS} / 10 \%(\mathrm{v} / \mathrm{v})$ Triton X-100. Delipidated serum was spiked with cholesteryl acetate stock to achieve concentrations of $0.5,1,3,5,7$ and $9 \mathrm{mM}$ total cholesterol (TC).

For method development, sensors were operated at $-100 \mathrm{mV}$ vs $\mathrm{Ag} / \mathrm{AgCl}$ pseudo-reference electrode using an Autolab PGSTAT128N with NOVA 1.6 software (Metrohm, UK) in time-based amperometric mode. Measurement involved an incubation period of $200 \mathrm{~s}$, followed by amperometric measurement for $240 \mathrm{~s}$. The concentration of cholesteryl acetate was correlated against the current at $240 \mathrm{~s}$. For operation of the SIMS device, incubation and amperometric measurement were combined into a single interval of $400 \mathrm{~s}$.

\section{RESULTS AND DISCUSSION}

Fig. 1 illustrates the complete twenty-two layer stack of the SIMS device. The development of the integrated sensor system involved the combination of multiple heterogeneous fabrication processes and associated materials including screen printing of metallic and non-metallic conductors, inkjet printing of functional electrocatalysts, enzymes and stabilizers, photolithography and lamination, to combine printed sensor, display and battery onto a single polyethylene naphthalate (PEN) substrate. The following sections detail the individual component characteristics and the operation of the integrated device in the measurement of $\mathrm{H}_{2} \mathrm{O}_{2}$ and total cholesterol (TC).

Printed electrochromic display. The display is a critical component of any diagnostic device as it represents the interface between the device and the user and must give clear and unambiguous information. Several types of printed display technologies have been developed including organic light emitting diodes (OLEDs), electroluminescence (EL) and electrochromics (EC). ${ }^{8,9}$ While OLEDs offer excellent performance for high quality, active matrix displays, their power requirements, lack of bistability and their need for organic thin film transistor back planes makes them unnecessarily complex and costly for this application. EL also requires the continuous application of 


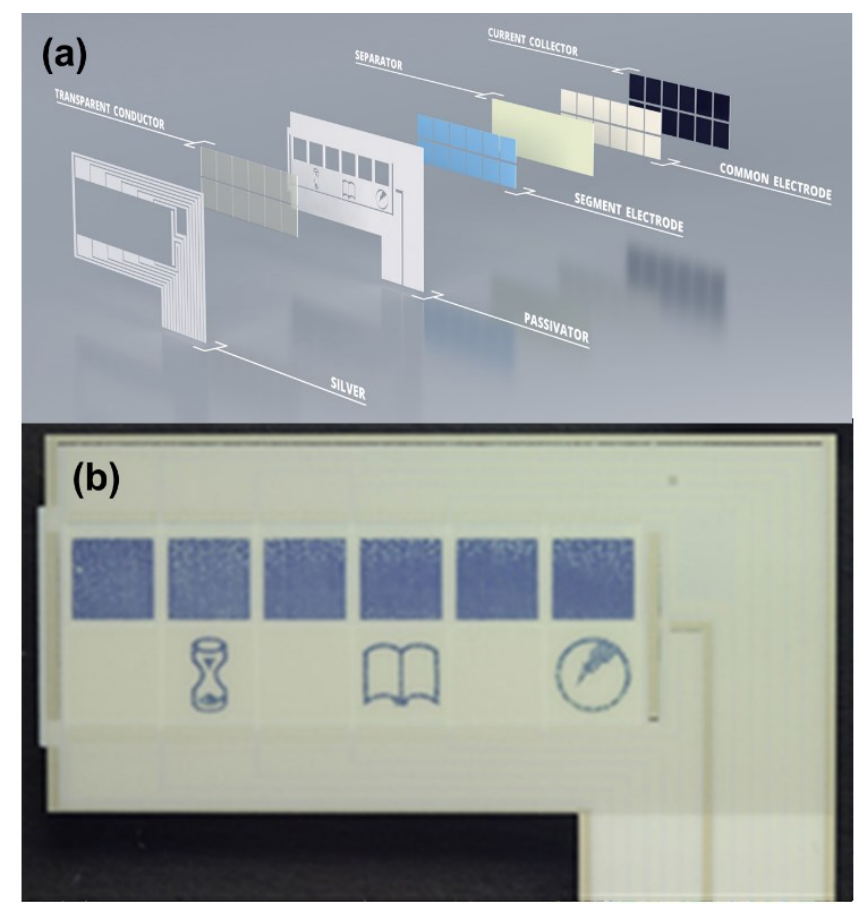

Figure 2. The printed electrochromic display. (a) Scheme of the seven layer display stack. (b) Layout of the electrochromic display showing upper bar display and lower power, hour glass and book icons to show operation, activity and reading of test result. power to remain illuminated. EC displays, however, undergo a (semi)permanent change in their redox characteristics due to the application of a potential. Viologens are bypyridyl derivatives which undergo a color change upon the application of an appropriate reduction potential. ${ }^{10,11}$

This change can be rendered bitable, requiring power only to switch on the display. This characteristic is particularly useful in single use applications such as diagnostic devices, with which the test result can be observed many hours or days after the test is performed. In this work, an EC display was developed using a fully screen-printed seven layer stack (Fig. 2a) onto a transparent ITO conductive substrate. First, the passivation layer was printed on the ITO substrate, followed by the silver conductor, segment electrode and separator as the next layers. The last three prints consisted of the common electrode, carbon electrode and electrolyte.

A non-numeric display was selected and designed which would indicate the level of the analyte being measured by activating an appropriate number of display bars (Fig. 2b). This semi-quantitative display approach was deemed to be the most effective way to allow interpretation by untrained lay users, while also greatly simplifying the design of the display driver aspect of the circuit. The display was also designed with additional functions including a power icon to indicate that the device was switched on and operational, an hour glass icon to indicate that the device was performing an operation and a book icon to indicate the reading of the result. Display elements with a size of $5 \mathrm{~mm}$ (14 point) were employed to facilitate clear and comfortable viewing by the user, allowing for a wide range of age and ability. ${ }^{12}$ Display icons were tested for the optimum drive voltage, current consumption and time to reach full intensity. It was found that display icons could be driven using a simple ac drive methodology with a nominal voltage of $1.1 \mathrm{~V}$ and a current consumption of $25 \mu \mathrm{A}$, reaching full intensity after 20 s.

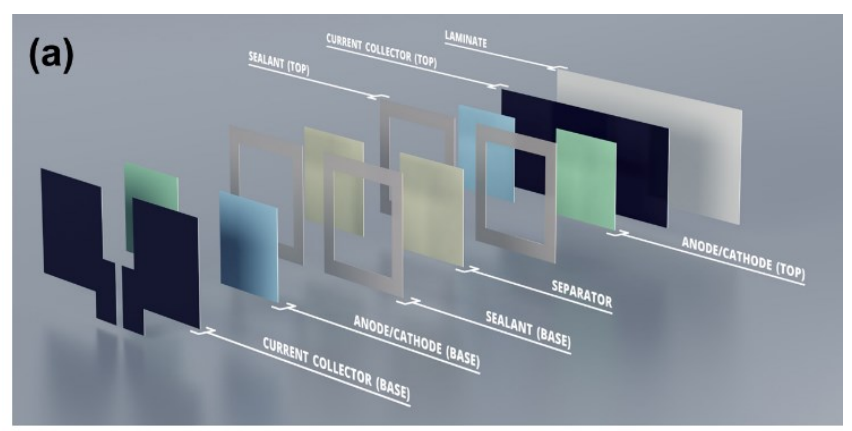

(b)

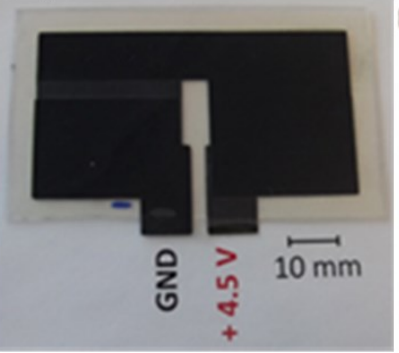

(c)
Ewe [M]

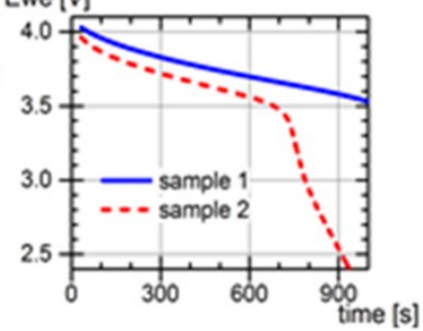

Figure 3. The printed battery. (a) Schematic of a two cell battery; (b) Photo of the three cell $4.5 \mathrm{~V}_{\text {nom }}$ battery. (c) Discharge curve at $1 \mathrm{~mA}$.

Printed battery. The printed battery employed in this system was primary cell based on zinc/manganese dioxide chemistry, designed for one-time (non-rechargeable) use. The nominal voltage of this chemical system for a single cell is $1.5 \mathrm{~V}$. These batteries can be manufactured by screen printing technology and can accommodate a range of operational voltages, currents and energy capacities by tailoring the geometries, numbers and configurations of cells employed. This battery concept and some applications have already been described. ${ }^{13-19}$

The battery was developed on a PEN foil substrate. A stacked, as opposed to a lateral battery configuration was selected as having greatest energy capacity, while also reducing cell footprint. This required electrodes to be printed on top of one another, with separators and electrolyte, with each half cell printed on a separate substrate, forming a sandwich and further sealed within a final PEN layer (Fig. 3a). The energy content of this chemical system is dependent on the amount of material within the battery cell. Screen printing was used to deposit layers of the required thickness in the range of 10 to $80 \mu \mathrm{m}$ to maximize energy capacity, while also maintaining a planar configuration.

One of the significant advantages of the screen printing process is that a series connection of batteries can be designed and easily manufactured. In the current setup, a series connection of three single cells was designed to deliver a voltage of $4.5 \mathrm{~V}_{\text {nom }}$ to drive conventional silicon circuitry (see Device operation) (Fig. 3b). The battery requirement for the system was defined as $>3 \mathrm{~V}$ with a current flow of $1 \mathrm{~mA}$ for up to $600 \mathrm{~s}$. The resulting battery was able to supply $>3.5 \mathrm{~V}$ for $1,000 \mathrm{~s}$ at a $1 \mathrm{~mA}$ discharge rate (Fig. 3c). The blue trace illustrates the typical performance of cells manufactured in this way, while the red trace illustrates batteries which occasionally fail early. However, they were still capable of driving the appropriate voltage for the duration of the assay.

Circuit design. The advent of the glucose monitor created a new paradigm of a two component system which was composed of a disposable printed sensor strip and a separate reusable electronic instrument incorporating battery, display and operational 
functions. However, with the advent of low cost, highly integrated silicon electronics using pick and place technology, and with rapid advances in organic electronics, the potential now exists to fully integrate the electronic circuit instrumentation with the rest of the device, giving rise to a new technological paradigm. However, silicon circuits must be produced in large volumes to achieve cost scaling and silicon costs can be reduced by increasing the numbers of devices on a wafer and by reducing their size and complexity. While there has also been significant progress in the development of organic circuit electronics, the potential for this technology to perform more complex processing tasks is still challenging. Thus, with the aim of facilitating the future integration of both silicon and organic electronics into a fully integrated diagnostic platform, a circuit design was proposed which was both simple but also fully capable of operating the proposed diagnostic device.

The organic circuit was designed with three amplifiers with a six-stage voltage converter, connected to the six-bit display element (Fig. 4a). The difference in resistance of the two electrochemical sensors is fed into the amplifiers and subsequently an output voltage is obtained. This voltage corresponds to the level of $\mathrm{H}_{2} \mathrm{O}_{2}$ or cholesterol in the sample based on the determination of a calibration slope. The voltage is subsequently fed into the voltage converter, which drives respective display elements. Table 1 indicates the levels TC and the respective sensor currents and drive voltages with the corresponding display element.

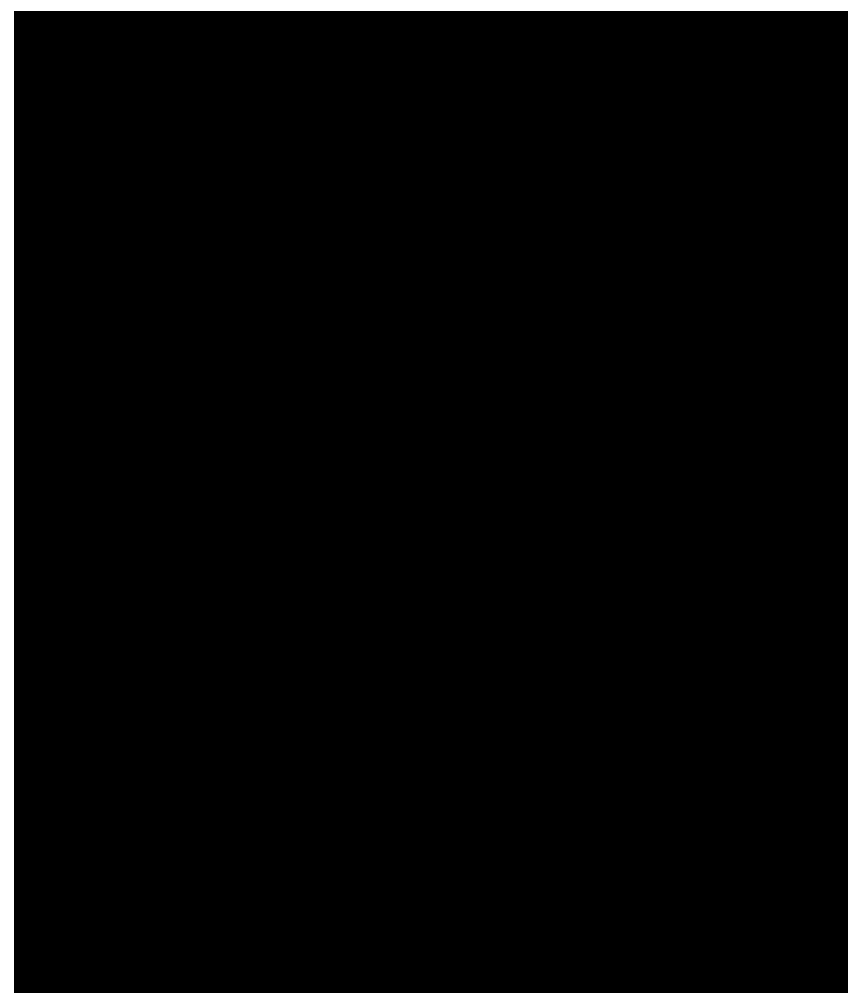

Figure 4. a) Block diagram of the organic circuit. Resistance measurements at the test (RT) and control (RC) sensors are amplified in a differential logic circuit and subtracted to yield a single amplified output voltage from the subtracting circuit. The voltage converter then drives the appropriate number of display bars. b) Block diagram of the equivalent silicon circuit. Sensors are driven via a digital to analogue converter (DAC). Outputs from the Test and Control sensors are amplified and a peripheral interface controller (PIC) performs subtraction and voltage conversion to output to the display driver. The display driver is composed of a bank of MOSFET switches. Display elements were driven at $1.2 \mathrm{~V}$.

Table 1. Relationships between cholesterol concentration, sensor currents, resistance ratios and display driver voltages for display illumination.

\begin{tabular}{lllll}
\hline $\begin{array}{l}\text { Display } \\
\text { bit }\end{array}$ & $\begin{array}{l}\text { Concentration } \\
(\mathrm{mM})\end{array}$ & $\begin{array}{l}\text { Current } \\
\left(10^{-7} \mathrm{~A}\right)\end{array}$ & $\begin{array}{l}\text { Voltage } \\
(\mathrm{V})\end{array}$ & $\mathrm{R}_{\mathrm{T}} / \mathrm{R}_{\mathrm{C}}{ }^{*}$ \\
\hline 0 & $>0$ & $<1.708$ & -3.19 & $<1$ \\
1 & $>2$ & $<1.838$ & -5.16 & $<0.535$ \\
2 & $>4$ & $<2.110$ & -7.19 & $<0.361$ \\
3 & $>5$ & $<2.244$ & -8.2 & $<0.311$ \\
4 & $>6$ & $<2.378$ & -9.21 & $<0.273$ \\
5 & $>7$ & $<2.512$ & -10.19 & $<0.244$
\end{tabular}

$* \mathrm{R}_{\mathrm{T}} / \mathrm{R}_{\mathrm{C}}$ : Resistance ratio of test and control electrodes.

A silicon circuit conforming to this simplified operational principle was fabricated using a conventional printed circuit board, and a conventional silicon integrated circuit (IC) was developed to facilitate testing of the printed sensor, display and battery. The integrated printed substrate was connected via a flexible parallel connector to the measurement board. The measurement circuit utilized a simple potentiostat configuration. ${ }^{20,21}$ The working electrode (WE) of the sensor was kept at the ground potential. The potential of the counter electrode (CE) was controlled by an operational amplifier so that the potential of the reference electrode (RE) in a steady state very closely follows the cell voltage $\left(\mathrm{V}_{\text {cell }}\right)$. The cell voltage $(-100 \mathrm{mV}$ in these measurements) was set by a digital-to-analog converter (DAC). The current flowing to the working electrode was converted to voltage by the transimpedance amplifier and digitized. A microcontroller unit (MCU) controlled the measurement process and output the responses to the printed display. Additional manual switching (on/off and measurement timing) were also included.

Printed electrochemical sensors. Electrochemical sensors are particularly suited to integration with organic electronics as they are, themselves electrical devices, do not require more costly or complex components such as is often the case in optoelectronics and they can also be easily manufactured in a twodimensional, planar configuration using print fabrication.

For the device demonstrated here, an amperometric sensor based on a novel electrocatalytic phenomenon which results in the enhanced electrocatalysis of $\mathrm{H}_{2} \mathrm{O}_{2}$ at a silver paste, screen printed electrode was employed. ${ }^{22}$ The novel electrocatalytic mechanism was based on the formation of liquid crystal lyotropic phases of surfactant which form on the surface of the silver and bring about an enhancement in the reduction of $\mathrm{H}_{2} \mathrm{O}_{2}$ at moderate reduction potentials (approx. $-100 \mathrm{mV}$ ) to avoid interferences. $\mathrm{H}_{2} \mathrm{O}_{2}$ is a very important molecule as it is a by-product of many chemical and biochemical reactions and so represents an important target for industrial and environmental monitoring and diagnostic device development. ${ }^{23-25}$ Previous work has demonstrated that a combination of dodecyl benzenesulfonic acid and $\mathrm{KCl}$ could bring about an up to 80 -fold increase in the reduction of $\mathrm{H}_{2} \mathrm{O}_{2}$ at silver-paste electrodes. It has also been demonstrated that this phenomenon occurred with other combinations of surfactant and salt, including Triton X-100 and $\mathrm{NaCl} .{ }^{26}$ and that they could be deposited using inkjet printing. ${ }^{27}$ 
Recently it was demonstrated that Triton X-100 can also be used to effectively solubilize lipoprotein-bound cholesterol and cholesterol esters in combination with this enhanced electrocatalysis to bring about the measurement of total cholesterol (TC). ${ }^{28}$ Here, we further demonstrate the full printed integration of a TC sensor with printed display and battery.
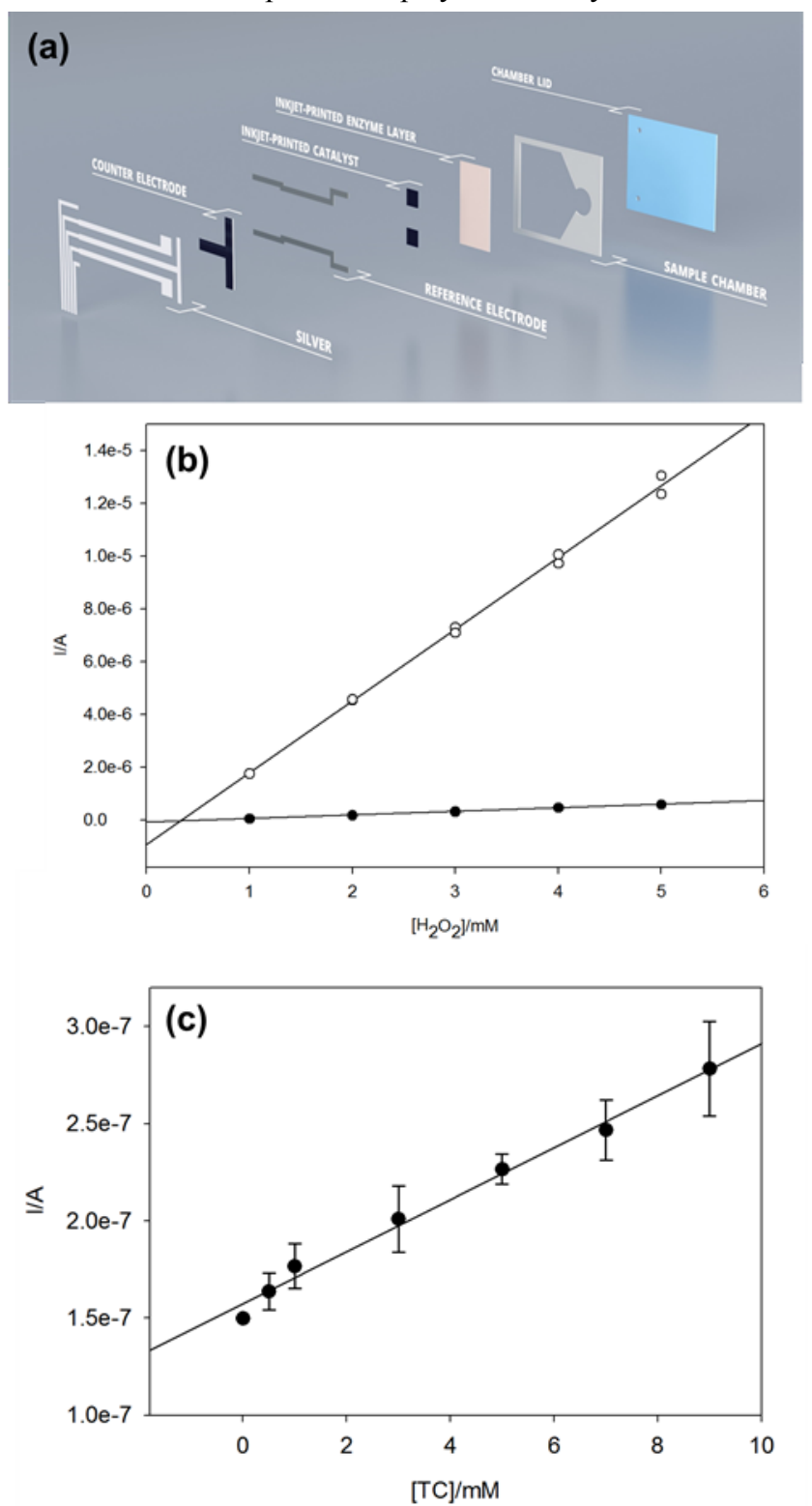

Figure 5. (a) Scheme of the electrochemical sensor. The sensor comprised a dual working electrode configuration with common counter and individual reference electrodes. A spacer and lid formed a microfluidic sample chamber of approx. $8 \mu \mathrm{L}$. The working electrodes were composed of screen-printed silver and modified with Triton X-100/PBS as catalyst. Total cholesterol measurement was achieved with the inkjet-printed deposition of cholesterol esterase, cholesterol oxidase and stabilizers. (b) Measurement of $\mathrm{H}_{2} \mathrm{O}_{2}$ in $0.5 \%$ Triton $\mathrm{X}-100,-0.1 \mathrm{~V}$ vs. $\mathrm{Ag} / \mathrm{AgCl}$, at $420 \mathrm{~s}$. Sensitivity $3.02 \times 10^{-5} \mathrm{~A} \cdot \mathrm{mM}^{-1} \cdot \mathrm{cm}^{-2}$ (c) Measurement of total cholesterol in serum. $1.34 \times 10^{-8}$ A. $\mathrm{mM}^{-1}$, $\mathrm{r}^{2}=0.99, \mathrm{RSD}<10 \%(\mathrm{n}=3)$.

The sensor electrodes (silver WE, silver/silver chloride RE and silver/carbon $\mathrm{CE}$ ) were fabricated using screen printing $\mathrm{A}$ dual electrode configuration was adopted, with one electrode providing measurement of background and the other measuring the reduction of $\mathrm{H}_{2} \mathrm{O}_{2}$ (Fig. 5a). This configuration was chosen to reduce the complexity of the circuit electronics and the calibration required in compensating for local device and environmental variations. Following inkjet printing of electrocatalyst and assay reagents, the sensor was encapsulated to form a thin layer microfluidic sample chamber requiring a sample volume of approx. $8 \mu \mathrm{L}$, to make it applicable to fingerstick blood draw measurements. $^{29}$

The measurement of $\mathrm{H}_{2} \mathrm{O}_{2}$ using electrodes modified with the inkjet-printed Triton X-100 $(0.5 \% \mathrm{v} / \mathrm{v})$ in PBS is shown in Fig. $5 \mathrm{~b}$. The fully printed sensor was tested for the measurement of $\mathrm{H}_{2} \mathrm{O}_{2}$ in the range of 0 to $5 \mathrm{mM}$. Catalysis was significantly enhanced in the presence of Triton X-100, while in its absence, little catalysis was observed, being $1.2 \times 10^{-5} \mathrm{~A}$ at $5 \mathrm{mM} \mathrm{H}_{2} \mathrm{O}_{2}$, in Triton $\left(3.02 \times 10^{-5}\right.$ A.mM $\left.{ }^{-1} . \mathrm{cm}^{-2}\right)$, compared with $5.91 \times 10^{-7} \mathrm{~A}$ in PBS alone; some 20-fold enhancement. This was comparable to the sensitivity of $4.9 \times 10^{-5} \mathrm{~A} \cdot \mathrm{mM}^{-1} \cdot \mathrm{cm}^{-2}$ previously achieved using inkjet-printed DBSA/KCl. ${ }^{27}$ Recently, $\mathrm{H}_{2} \mathrm{O}_{2}$ sensors based on inkjet-printed Prussian blue nanoparticles have achieved sensitivities of $7.62 \times 10^{-5}$ A. $\mathrm{mM}^{-1} \cdot \mathrm{cm}^{-2} .{ }^{30}$

For the measurement of TC, both ChEs and ChOx were deposited using inkjet printing, along with Triton X-100, and enzyme stabilizers. Measurement of cholesteryl ester in the range of 0 to $9 \mathrm{mM}$ was selected to demonstrate direct (undiluted) measurement of cholesterol in the clinically relevant range. ${ }^{30,31}$ The majority of cholesterol is transported by lipoproteins in the form of cholesteryl esters. These can be transformed to cholesterol by ChEs and to cholest-4-en-3-one and $\mathrm{H}_{2} \mathrm{O}_{2}$ by ChOx. Triton X-100 between 0.2 and $0.5 \%$ has been shown to be optimal for full release of cholesterol esters from both HDL and LDL, which is a pre-requisite for TC measurement. Other combinations of surfactant and protecting agents have been shown to result in selectivity to either LDL-C or HDL-C. ${ }^{28}$

The measurement of TC in the fully printed sensor device demonstrated good linearity in the range from 0 to $9 \mathrm{mM}$ with a slope of $1.34 \times 10^{-8}$ A.mM ${ }^{-1}, r^{2}=0.99, \mathrm{RSD}<10 \%(\mathrm{n}=3)$ (Fig. $5 \mathrm{c})$. Replicate measurements were made with different sensors with the same stock of TC standard. Levels of TC below 5.2 $\mathrm{mM}$ are considered desirable and of low risk while 5.2 to 6.2 $\mathrm{mM}$ is considered borderline high risk. Values greater than 6.2 $\mathrm{mM}$ are considered high risk. This was reflected in display colour coding of green ( 0 to $4 \mathrm{mM}$ ), yellow ( 4 to $5 \mathrm{mM}$ ), orange ( 5 to $6 \mathrm{mM})$, light red $(6$ to $7 \mathrm{mM})$ and dark red $(>7 \mathrm{mM})$. The resulting sensor was capable of measuring TC from 0 to $9 \mathrm{mM}$ at moderate $(-100 \mathrm{mV})$ potentials to minimize potential redox interferences. The presence of surfactant may also assist in reducing interference due to biofouling. The generation of submicroampere currents, resulting in $\mathrm{nW}$ levels of power consumption is an important feature in minimizing power requirements (battery size).

Fabrication integration. Organic and printed electronics presents an exciting, but also a challenging opportunity to mass manufacture novel and advanced devices. The physical integration of multiple functionalities creates an exponential complexity problem due to the numbers of different functional materials, their particular process requirements and their compatibility with other materials and other processes. As a result, achieving true integration of such heterogeneous devices has remained challenging. Various approaches have been taken to address this problem. Some have used multiple substrates on which to fabricate various system elements ${ }^{33}$, while others have used prefabricated sub-components such as separate sensor strips and 
battery components to achieve instrument-free glucose measurement. ${ }^{33}$ However, the approach taken here was to demonstrate integration of heterogeneous subsystems on a single substrate, for which all processes must be made fully inter-compatible at the process level. Several integration principles were applied here to achieve integration. Redundancy was removed from the various components, where possible. Processes were also sequenced to ensure that materials with least sensitivity, or more rigorous process requirements were performed prior to those with greater sensitivity. The design layout was also optimized to eliminate any potential impact of one subsystem on another. The integrated printed sensor, battery and display is illustrated in Fig. 6. As the display is printed 'face down' to the substrate and the sensor is fabricated 'face up' from the substrate, a simple microfluidic was incorporated to allow sample application on the same side as the display. Sensor and display were fabricated together on a single substrate and the battery was prepared on a separate substrate and subsequently integrated onto the common substrate. Final addition of sensor modification layers was then performed via inkjet printing, resulting in a layer stack of 22 processing steps. Although only 24 fully integrated devices were fabricated, operational yields of $100 \%$ were achieved by having good design tolerances in all print process steps.

Device operation. The integrated prototype device is shown in Fig. 7a. The device was activated by operating a manual on/off touch switch which activates the circuit and illuminates the power on icon. The sample chamber was composed of a hydrophilic polymer bonded with a PSA. Filling was by capillary action, with air escape through two vias in the lid of the sample chamber. Upon addition of sample $\left(8 \mu \mathrm{L}\right.$ of $\mathrm{H}_{2} \mathrm{O}_{2}$ or cholesterol in serum), the second switch is activated to begin the measurement timing of the device. A potential of $-100 \mathrm{mV}$ is applied to the sensor working electrodes of both the control and test cells. For measurement of TC, the assay employed a $400 \mathrm{~s}$ incubation between cholesterol and cholesterol oxidase to allow full conversion to cholest-4-en-3-one and hydrogen peroxide. During this period, the hour glass icon is illuminated. After $400 \mathrm{~s}$, the difference in current between the two sensors is subtracted and applied to the stored calibration constant of the device and illuminates the appropriate number of display bars.

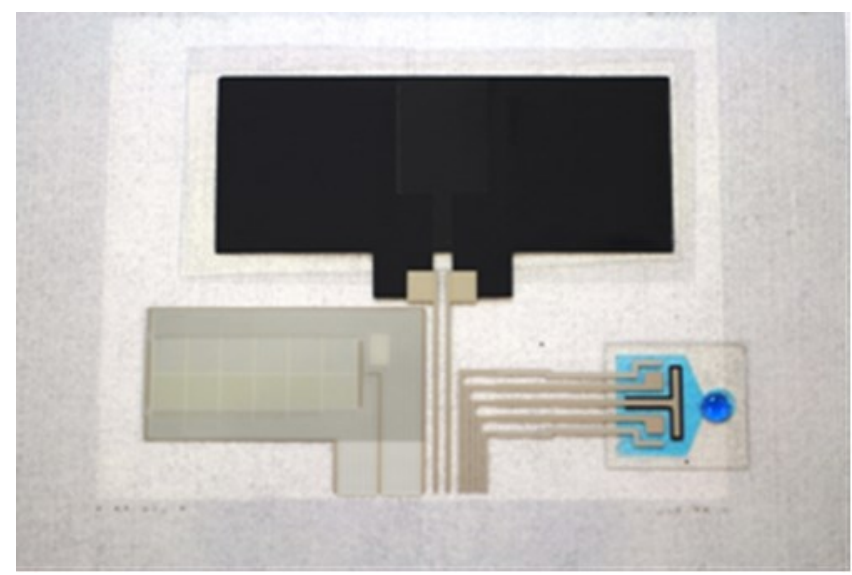

Figure 6. Integrated printed system components. Sensor (bottom right), display (bottom left) and battery (top) were effectively integrated onto a single substrate using a combination of screen printing, inkjet printing, bonding and lamination processes into a monolithic 22 layer stack.
Fig. $7 \mathrm{~b}$ shows the test set up of separate display and sensor to allow reuse of the displays during laboratory testing. To the right of Fig. 7b shows the illumination of one display bar for 1 $\mathrm{mM} \mathrm{H}_{2} \mathrm{O}_{2}$, three bars for $3 \mathrm{mM}$ and five bars for $5 \mathrm{mM}$. In addition, the 'book' icon is displayed indicating that it is time for the device reading. A video of the operation of the device can be viewed in Supporting Information.

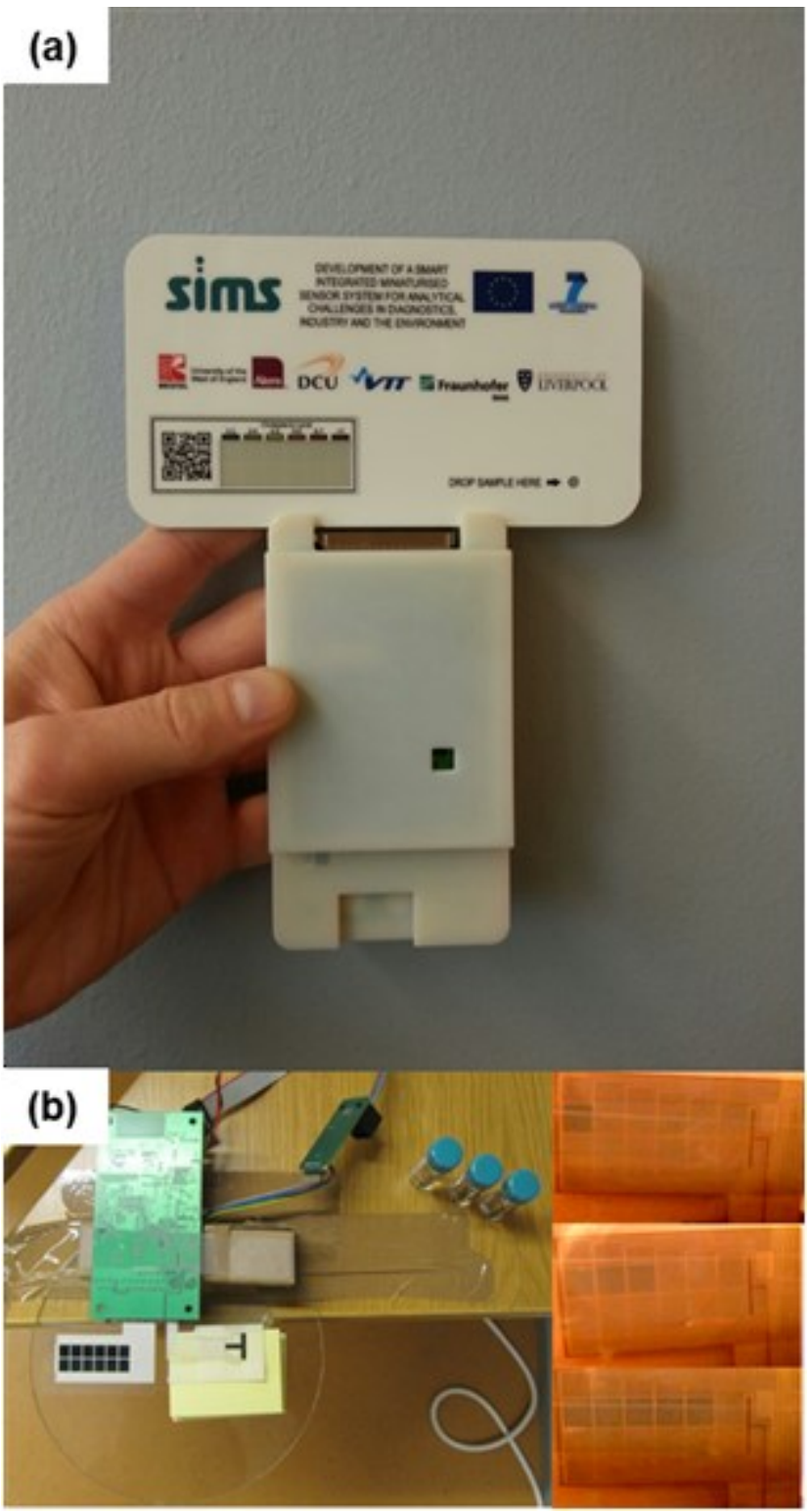

Figure 7. Operation of the integrated sensor system. (a) The assembled prototype showing printed cover with QR code, cholesterol concentration values and sample application zone. Below is the electronic control board. (b) Testing of the device using separate displays and sensors (displays could be reused during laboratory testing). On the right shows the results of the addition of 1,3 and 5 $\mathrm{mM} \mathrm{H} \mathrm{H}_{2} \mathrm{O}_{2}$, resulting in the activation of an equivalent number of display blocks.

Communications interface. As well as being readable visually, a communications interface was also developed in association with the device which allows remote transmission of the test result via a mobile phone (Fig. 8). This interface was supported with additional printed features around the display (Fig. 7a). These include a color-coded bar to indicate to the user 
whether the measured level of analyte (in this instance cholesterol) was normal or too high. In addition, the device was designed with QR coding. This coding contains specific device identification that can be used in several ways including device authentication, supply chain management and quality control monitoring. ${ }^{32}$

In addition, the QR also allows the location and orientation of the display to be detected. Result detection and interpretation is performed using an Android smartphone application. The 'app' is designed to provide patient identification and authentication. The app employed the phone's camera to take a picture of the display. Using the QR code, the app identifies the device, locates the device display, detects the location and number of display blocks activated, and can record the date, time and location of the test. A picture of the display, along with test result and associated metadata can be automatically sent via SMS to a remote, healthcare management site to monitor health status, treatment efficacy and testing compliance. The issue as to whether or not the test result should be interpreted locally by the phone has implications for the type of medical device regulatory approval required of the mobile phone. Operation of the mobile phone communications app can be seen in the accompanying video (Supporting Information).

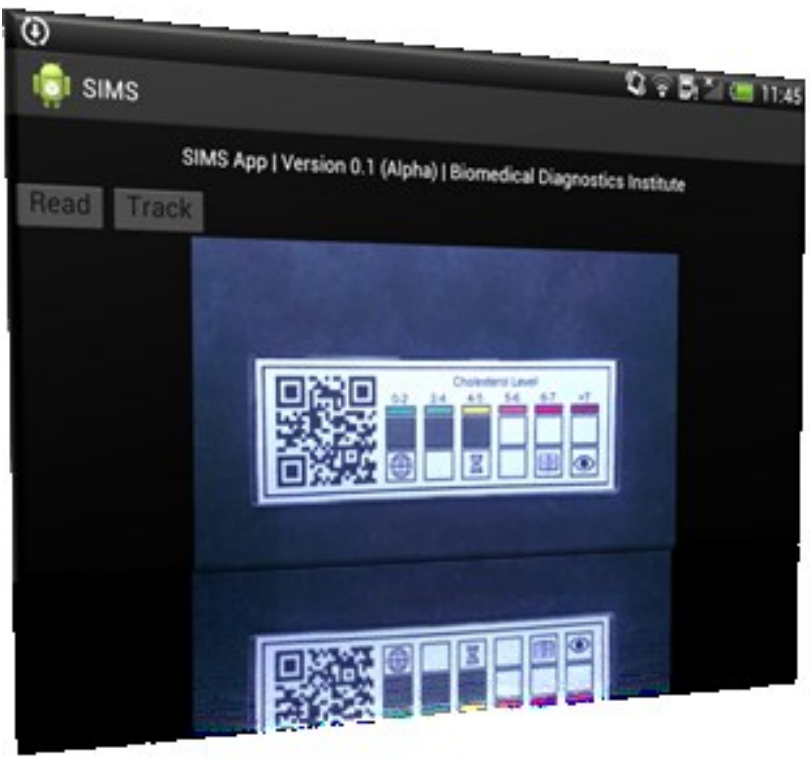

Figure 8. The communications interface mobile phone application. The app allows an image of the SIMS device to be captured by the phone's camera. Image processing algorithms automatically locate the chip and the QR features. The reading on the chip is automatically recorded (small grey squares) and the QR codes are used to identify the test and track the results.

Cholesterol testing is currently used to manage hypercholesterolemia which is a major risk factor in atherosclerosis. Treatment is typically by way of lifestyle management (diet and exercise), as well as administration of statins. Point of care devices employing strips and meters are available and strips typically retail for between US\$2 and US\$3 per strip. This requires the more challenging acceptance and integration of such technology into the lifestyle of often elderly patients. Pharmacy-based testing is also available at over US\$12 per test. The prototype production cost of the SIMS device was approximately US\$5, which could be significantly reduced during production scale up and mass manufacture to approximately US\$1.50 or less, with a target sale price of US\$5. It also offers users greater simplicity of self-testing, as well as connectivity with health care providers. Current test time is approximately seven minutes which has the potential to be reduced to less than two or three minutes and which would be acceptable to most users. While the measurement of TC has been demonstrated here, the addition of a sensor for the measurement of high density lipoprotein cholesterol (HDL-C) would facilitate subtractive measurement of non-HDL-C (low density lipoprotein or LDL and very low density lipoprotein or VLDL cholesterol) using the Friedwald equation. ${ }^{35}$ Non-HDL-C is considered to be atherogenic and so is better than the separate measurement of LDL-C. ${ }^{36}$

\section{CONCLUSIONS}

Printed electronics was successfully used to achieve the fabrication of a sensor, display and battery into a fully integrated diagnostic device. The sensors were shown to be capable of the quantitative measurement of $\mathrm{H}_{2} \mathrm{O}_{2}$ and total cholesterol, while the display, battery and circuit all demonstrated component functionality. The final integrated device was also able to demonstrate the measurement of $\mathrm{H}_{2} \mathrm{O}_{2}$. The device was also capable of remote result transmission via mobile phone. Such a device could form the basis of a new approach to diagnostic device fabrication and enhance the management of disorders such as hypercholesterolemia.

\section{ASSOCIATED CONTENT}

\section{Supporting Information}

The Supporting Information is available free of charge on the ACS Publications website.

Video showing operation of the SIMS device (.md4)

Video showing operation of the mobile phone interface (.avi)

\section{AUTHOR INFORMATION}

\author{
Corresponding Author \\ * Email: tony.killard@uwe.ac.uk; Tel.: +44(0)117 3282147.
}

\section{Author Contributions}

The manuscript was written through contributions of all authors. All authors have given approval to the final version of the manuscript. $\$$ These authors contributed equally.

\section{Notes}

The authors declare no conflicts of interest.

\section{ACKNOWLEDGMENT}

The authors would like to acknowledge the financial assistance of EU, FP7/2007-2013, under grant number 257372.

\section{REFERENCES}

(1) Kamyshny, A.; Magdassi, S. Small. 2014, 10, 3515-3535.

(2) Park, S.; Vosguerichian, M; Bao, Z. Nanoscale, 2013, 5, 1727 1752.

(3) Chang, J.; Ge, T.; Sanchez-Sinencio, E. Circuits and Systems (MWSCAS), 2012 IEEE 55th International Midwest Symposium on, 2012, 582-585.

(4) Lupo, D.; Clemens W.; Breitung S.; Hecker K. in Applications of Organic and Printed Electronics, ed. E. Cantatore, Springer, New York, 2013, pp. 1-26. 
(5) Singh, M.; Haverinen, H. M.; Dhagat, P.; Jabbour, G. E. Adv. Mater. 2010, 22, 673-685.

(6) Newman, J. D.; Turner A. P. F. Biosens. Bioelectron. 2005, 20 , 2435-2453.

(7) Wang, J. Chem. Rev. 2008, 108, 814-825.

(8) Carpi F.; De Rossi, D. Optics Laser Technol. 2006, 38, 292-305.

(9) Yang, Y.; Chang, S.-C.; Bharathan, J.; Liu, J. J. Mater. Sci. Mater. Electron. 2000, 11, 89-96.

(10) Ryu, J.-H.; Lee, J.-H.; Han, S.-J.; Suh, K.-D. Macromol. Rapid Commun. 2006, 27, 1156-1161.

(11) Bonhôte, P.; Gogniat, E.; Campus, F.; Walder, L.; Grätzel M. Displays, 1999, 20, 137-144.

(12) Redish, J.; Chisnell, D. AARP, Washington, 2004, pp. 1-64.

(13) Willert, A.; Kreutzer, A.; Geyer, U.; Baumann, R. R. in European Conference and Exhibition on Integration Issues of Miniaturized Systems - MEMS, MOEMS, ICs and Electronic Components, Brussels, 2009.

(14) Espig, M.; Siegel, F.; Hammerschmidt, J.; Willert, A.; Baumann, R. R. in 28th International Conference on Digital Printing Technologies/Digital Fabrication 2012, Quebec, Canada, 2012, pp. 168170 .

(15) Willert, A.; Espig, M.; Sowade, E.; Baumann, R. R. in $7^{\text {th }}$ International Conference \& Exhibition on Integration Issues of Miniaturized Systems - MEMS, NEMS, ICs and Electronic Components, Amsterdam, 2013.

(16) Willert, A. IEEE 2013 International Semiconductor Conference Dresden - Grenoble (ISCDG), Dresden, Germany; September 26-27, 2013. URL: http://iscdg.org/program/.

(17) Willert, A.; Espig, M.; Sowade, E.; Baumann, R. R. Proceedings of Smart Systems Integration 2013, Amsterdam, The Netherlands, March 13-14, 2013. ISBN: 978-3-8007-3490-0 URL: http://www.mesago.de/en/143ssi/The_conference/Program/2479_program_detail.htm. VDE VERLAG GMBH Berlin Offenbach.

(18) Willert, A.; Helmert, M.; Baumann, R. R. Smart Systems Integration, Munich, Germany, 2016, ISBN 978-3-95735-040-4

(19) Willert, A.; Helmert, M.; Baumann, R. R. ICFPE, Yamagata 2016, Japan.
(20) Kissinger P. T.; Heineman, W. R. Laboratory Techniques in Electroanalytical Chemistry, Marcel Dekker, New York, NY, USA, 1996.

(21) Ahmadi M. M.; Jullien, G. A. IEEE Trans. Circ. Sys. I, 2009, $56,1339-1348$

(22) Gonzalez-Macia, L.; Smyth, M. R.; Morrin, A.; Killard, A. J. Electrochim. Acta, 2011, 56, 4146-4153.

(23) Chen, W.; Cai, S.; Ren, Q.-Q.; Wen, W.; Zhao, Y.-D. Analyst, 2012, 137, 49-58.

(24) Guascito, M. R.; Filippo, E.; Malitesta, C.; Manno, D.; Serra, A.; Turco, A. Biosens. Bioelectron. 2008, 24, 1057-1063.

(25) Zhang Y.; Wilson, G. S. J. Electroanal. Chem. 1993, 345, 253271.

(26) Gonzalez-Macia, L.; Smyth, M. R.; Morrin A.; Killard, A. J. Electrochim. Acta, 2011, 58, 562-570.

(27) Gonzalez-Macia, L.; Smyth M. R.; Killard, A. J. Electroanal. 2012, 24, 609-614.

(28) Ahmadraji T.; Killard. A. J. Anal. Meth., 2016, 8, 2743-2749.

(29) Gubala, V.; Harris, L. F.; Ricco, A. J.; Tan, M. X.; Williams, D. E. Anal. Chem. 2012, 84, 487-515.

(30) Cinti, S.; Arduini, F.; Moscone, D.; Palleschi, G.; Killard. A.J. Sensors 2014, 14, 14222-14234.

(30) Zhao, C.; Wan, L.; Jiang, L.; Wang, Q.; Jiao, K. Anal. Biochem. 2008, 383, 25-30.

(31) Naito, H. K.; Hutchison, J. D.; Bowers, G. N.; Baillie, E. E.; Joseph, J. M.; Myers, G. L.; Hainline, A.; Cooper, G. R. ;Seckinger, D.; Ross, J. W.; Aziz, K.; Danniel, A.; Schaffer, R.; Vanderlinde, R. E.; Bachorik, P.; Hartmann, A.; Kessler, G.; LaRosa, J. C.; Statland, B. E.; Warnick, R.; Cleeman, J. I.; Lippel, K.; Rifkind, B. Clin. Chem. 1988, 34, 193-201.

(32) Quintero, A.V.; Van Remoortere, B.; Smits, E.C.P.; van den Brand, J.; Briand, D.; Schoo, H.F.M.; Rooij, N.F.; Microelectron. Eng., 2013, 110, 52-58.

(33) Beni, V.; Nilsson, D.; Arven, P.; Norberg, P.; Gustafsson, G.; Turner, A.P.F. ECS J. Solid State Sci. Technol., 2015, 4, S3001-S3005.

(34) Seenivasagam V.; Velumani, R. Comput. Math. Methods Med. 2013, 2013, 516465.

(35) Friedewald, W. T. Clin. Chem. 1972, 18, 499-502.

(36) Ahmadraji, T. Killard, A.J. Anal. Meth. 2013, 5, 3612-3625. 


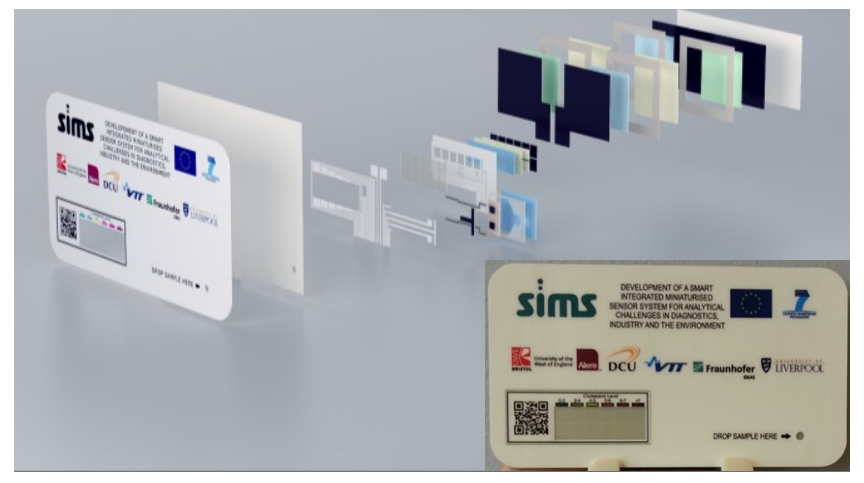

